\title{
The Time Course of Markers of Neutrophil Extracellular Traps in Patients Undergoing Revascularisation for Acute Myocardial Infarction or Stable Angina Pectoris
}

\author{
Ragnhild Helseth, ${ }^{1,2}$ Svein Solheim, ${ }^{1}$ Harald Arnesen, ${ }^{1,2}$ \\ Ingebjørg Seljeflot, ${ }^{1,2}$ and Trine Baur Opstad ${ }^{1,2}$ \\ ${ }^{1}$ Center for Clinical Heart Research, Department of Cardiology, Oslo University Hospital Ullevål, Oslo, Norway \\ ${ }^{2}$ Faculty of Medicine, University of Oslo, Oslo, Norway
}

Correspondence should be addressed to Ragnhild Helseth; ragnhild.helseth@gmail.com

Received 11 August 2016; Revised 5 November 2016; Accepted 15 November 2016

Academic Editor: Veronica Tisato

Copyright ( 2016 Ragnhild Helseth et al. This is an open access article distributed under the Creative Commons Attribution License, which permits unrestricted use, distribution, and reproduction in any medium, provided the original work is properly cited.

\begin{abstract}
Background and Aims. Neutrophil extracellular traps (NETs) have been identified in acute myocardial infarction. We assessed the time profile and association with infarct size for NETs markers in ST-elevation myocardial infarction (STEMI) and stable angina pectoris (AP). Methods. In 20 patients with STEMI and 10 with AP undergoing percutaneous coronary intervention (PCI), blood samples were collected before PCI (only AP group) and after 3 and 12 hours, days 1, 3, 5, 7, and 14 for measurement of NETs markers. Results. Double-stranded deoxyribonucleic acid (dsDNA) and nucleosome levels were higher in STEMI than AP until day 3 and 12 hours ( $p<0.03$, all). DsDNA declined after day 5 in both groups $(p<0.04$, all), while nucleosomes declined until day 3 only in the AP group $(p<0.05$, all). DsDNA correlated with peak troponin T and creatine kinase MB (CKMB) at day $5(r=0.48, p=0.03$, both) and with MRI-measured infarct size at days 5 and $7(r=0.61, p=0.01$ and $r=0.52, p=0.04$, resp.), while nucleosomes correlated with infarct size at day $5(r=0.58, p=0.02)$. Conclusions. High levels of NETs markers were observed in STEMI shortly after revascularisation and were partly associated with infarct size. The decline thereafter in both groups indicates a role for NETs in both acute and chronic atherothrombosis.
\end{abstract}

\section{Introduction}

Neutrophil cell activation in acute myocardial infarction (MI) has lately gained attention. Quantitative amounts of neutrophils and several neutrophil granule proteins are suggested to be predictive of infarct size, left ventricular ejection fraction (LVEF), new cardiovascular events, and death after acute MI [1-6]. A decade ago, it became evident that neutrophils upon activation are able to release parts of their nuclear content with residing neutrophil granule proteins into the extracellular space to form spindle-like networks called neutrophil extracellular traps (NETs) [7]. Although NETs initially were thought to have their main role in infectious diseases, ensuring entrapment of microorganisms in areas with high concentrations of antimicrobial proteins [7], NETs have lately been identified in coronary artery disease (CAD) [8-10].
In acute MI, high levels of circulating cell-free deoxyribonucleic acid (DNA), a surrogate marker of NETs, have been reported [11-15] and also linked to infarct size $[8,13,15]$. NETs are also suggested to be present in coronary thrombi $[8,9]$, in mural atherosclerotic plaques $[16,17]$, and in stable angina pectoris (AP) where they further seem to serve as a predictor of coronary artery severity and risk of new coronary events [10]. By the seemingly wide-ranging prothrombotic properties like platelet entrapment and activation [18], activation of the coagulation system $[19,20]$, and inhibition of fibrinolysis [21], NETs are potentially important players in the pathogenesis of atherothrombosis.

The dynamic profile of circulating NETs in the acute and subacute phase of ST-elevation MI (STEMI) has not been reported in detail previously. In this study, we aimed to explore the time profile of the circulating surrogate markers of NETs double-stranded deoxyribonucleic acid 
(dsDNA) and nucleosomes (DNA-histone complexes) in patients with STEMI or stable AP undergoing coronary angiography with percutaneous coronary intervention (PCI). The time profiles of myeloperoxidase (MPO) and pentraxin 3 (PTX3), well-known neutrophil cell granule proteins, were also investigated in order to explore whether these proteins could be reflected in the measured markers of NETs. We further assessed whether levels of dsDNA, nucleosomes, and MPO were related to indices of myocardial injury and left ventricular function.

\section{Material and Methods}

2.1. Study Design. Thirty patients with CAD, 20 with STEMI, and 10 with stable AP, admitted to Oslo University Hospital (OUS) Ullevål, Norway, undergoing successful revascularisation with PCI, were included. Details of the study design have been reported previously [22]. In brief, inclusion criteria in the STEMI group were characteristic clinical symptoms, electrocardiographic ST-elevations, and angiographic verification of coronary artery occlusion, while characteristic clinical symptoms and CAD angiographically suitable for PCI were inclusion criteria in the AP group. Exclusion criteria in both groups were previous transmural infarction, cardiogenic shock, and serious comorbidity. All patients were medically treated according to current guidelines and gave written informed consent for study participation. The study protocol was approved by the Regional Committee for Medical Research Ethics and conforms to the ethical guidelines of 1975 Declaration of Helsinki.

2.2. Blood Sampling. Blood samples were collected by standard venipuncture immediately before PCI in the AP group and after 3 and 12 hours, days 1, 3, 5, 7, and 14 in both groups. All blood samples from day 1 and further were obtained in fasting state before intake of morning medication. Routine analyses were obtained by conventional methods. Serum was prepared by centrifugation within 1 hour at $2500 \times \mathrm{g}$ for $10 \mathrm{~min}$ and EDTA plasma was prepared by centrifugation within 1 hour at $2500 \times \mathrm{g}$ for 20 minutes at $4^{\circ} \mathrm{C}$, both stored at $-80^{\circ} \mathrm{C}$ until analysed.

2.3. Laboratory Analyses. Levels of dsDNA and nucleosomes were quantified in serum by use of Quant-iT Picogreen dsDNA Assay \# P11496 (Invitrogen, Carlsbad, CA, USA) and ELISA Cell Death Detection kit \# 11774425001 (Roche Diagnostics, Indianapolis, USA), respectively. Optical density values in the nucleosome assay were normalized to an internal positive control and expressed as arbitrary units of nucleosomes per milliliter $(\mathrm{NU} / \mathrm{mL})$. All related samples from one patient and samples from both groups were analysed on the same plate in order to reduce the significance of interassay variability. Levels of MPO were measured in EDTA plasma by ELISA (Mercodia AB, Uppsala, Sweden). Interassay coefficient of variation $(\mathrm{CV})$ for the measurements of dsDNA, nucleosomes, and MPO were $2.5 \%, 28.7 \%$, and $6.3 \%$, respectively.
Myocardial injury was assessed by peak serum levels of troponin $\mathrm{T}$ (reference value $<0.03 \mu \mathrm{g} / \mathrm{L}$ ) and creatine kinase $\mathrm{MB}(\mathrm{CKMB})$ (reference value $<5 \mu \mathrm{g} / \mathrm{L}$ ) and by gadolinium late contrast enhancement technique based infarct size (\%) measured by magnetic resonance imaging (MRI) after 6 weeks. Left ventricular function was assessed by left ventricular ejection fraction (LVEF) by MRI after 6 weeks. MRI measures were available in 16 patients.

2.4. Statistical Analyses. As the majority of variables were skewly distributed, nonparametric statistics were used throughout. Group differences were assessed by MannWhitney $U$ Test or Fisher's Exact Test as appropriate, while overall change within a group and change between two time points within a group were assessed by Friedman Test and Wilcoxon Signed Rank Test, respectively. Correlation analyses were performed by Spearman rho. Due to the hypothesis generating nature of the study, Bonferroni corrections for multiple comparisons were not performed. The level of significance was set to $p<0.05$. All statistical analyses were performed by SPSS software package, version 23.0.

\section{Results}

Patient characteristics at baseline are shown in Table 1. Beyond more established CAD in the stable AP group and thus more frequent use of secondary prophylactic cardiovascular drugs, the groups were comparable.

\subsection{The Time Profile of Circulating Markers of NETs}

3.1.1. Double-Stranded Deoxyribonucleic Acid (dsDNA). Levels of dsDNA were significantly higher in the STEMI group than in the AP group at all time points until day $3(p<0.03$ for all) (Figure 1(a)).

Within both groups, a significant overall change in dsDNA levels was observed ( $p \leq 0.02$ ), with values declining from 3 hours in the STEMI group and from baseline in the stable AP group, respectively, to day 5 and all later time points $(p<0.04$ for all) (Figure 1(a)).

3.1.2. Nucleosomes (DNA-Histone Complexes). Levels of nucleosomes were significantly higher in the STEMI group compared to the stable AP group at 3 and 12 hours $(p<0.03$ for both) (Figure 1(b)).

No significant overall change or change from 3 hours to later time points was observed in the STEMI group. In the stable AP group, levels declined from baseline until day 3 ( $p<0.05$ for all), although no significant overall change was observed (Figure 1(b)).

3.2. The Time Profile of MPO. No difference between the groups was observed for levels of MPO at any time point (Figure 1(c)).

In both groups, a significant overall change was observed $(p<0.01)$ with declining levels from 3 hours to all later time points $(p<0.01$ for all). Within the stable AP group, 
TABLE 1: Clinical characteristics of the study population.

\begin{tabular}{|c|c|c|c|}
\hline & $\begin{array}{c}\text { Acute } \\
\text { MI group } \\
n=20\end{array}$ & $\begin{array}{c}\text { Stable } \\
\text { AP group } \\
n=10\end{array}$ & $p$ \\
\hline Age (yrs) & $60(54-68)$ & $64(54-71)$ & ns \\
\hline Female gender & 5 & 1 & ns \\
\hline Established CAD & 0 & 7 & $<0.01$ \\
\hline Hypertension & 7 & 4 & ns \\
\hline Diabetes mellitus (type 1 or 2 ) & 2 & 2 & ns \\
\hline Previous or current smoking & 10 & 3 & ns \\
\hline \multicolumn{4}{|l|}{ Medication at study inclusion } \\
\hline Acetylsalicylic acid & 1 & 7 & $<0.01$ \\
\hline Clopidogrel & 0 & 0 & ns \\
\hline ACE/AT II antagonists & 5 & 2 & ns \\
\hline Beta-blocker & 2 & 4 & 0.02 \\
\hline Aldosterone antagonist & 0 & 0 & ns \\
\hline Insulin & 0 & 0 & ns \\
\hline Diuretics & 0 & 0 & ns \\
\hline Statins & 1 & 7 & $<0.01$ \\
\hline \multicolumn{4}{|l|}{ Medication at hospital discharge } \\
\hline Acetylsalicylic acid & 20 & 10 & ns \\
\hline Clopidogrel & 20 & 9 & ns \\
\hline ACE/AT II antagonists & 11 & 2 & ns \\
\hline Beta-blocker & 18 & 6 & ns \\
\hline Aldosterone antagonist & 0 & 0 & ns \\
\hline Insulin & 0 & 0 & ns \\
\hline Diuretics & 1 & 0 & ns \\
\hline Statins & 20 & 10 & ns \\
\hline
\end{tabular}

\begin{tabular}{lc}
\hline Indices of infarct size and left ventricular function & \\
Peak troponin T $(\mu \mathrm{g} / \mathrm{L})$ & $3.8(2.1-6.1)$ \\
Peak CKMB $(\mu \mathrm{g} / \mathrm{L})$ & $158(93-268)$ \\
Infarct size $(\mathrm{MRI}, \%)^{\mathrm{a}}$ & $6.6(3.2-10.7)$ \\
LVEF $(\mathrm{MRI}, \%)^{\mathrm{a}}$ & $58(53-66)$ \\
\hline
\end{tabular}

Values are given as numbers or medians (25-75 percentiles) unless otherwise stated. CAD: coronary artery disease, defined as previous angina, $Q-$ or non- $Q$ infarction, percutaneous intervention (PCI), or coronary artery bypass grafting (CABG). ACE/AT II antagonists: angiotensin converting enzyme/angiotensin II antagonists. CKMB: creatine kinase MB. LVEF: left ventricular ejection fraction. ${ }^{\mathrm{a}}$ Measured 6 weeks after study inclusion.

a significant increase from baseline to 3 hours was observed, sustained elevated at days $1,3,5$, and 7 ( $p \leq 0.03$ for all) (Figure 1(c)).

\subsection{Correlations between Markers of NETs and Neutrophil} Proteins. In the total cohort, dsDNA and nucleosome levels intercorrelated significantly at the majority of time points (Table 2). Levels of MPO did not correlate with either dsDNA or nucleosome levels beyond a negative correlation to nucleosomes at 3 hours $(r=-0.43, p=0.02)$. Previously, we have investigated the time profile of pentraxin 3 (PTX3), another neutrophil granule protein and thus potential NETs component in the same cohort and observed that PTX3 levels were elevated shortly after PCI [23]. PTX3 levels did not
TABLE 2: Correlation between levels of dsDNA and nucleosomes at corresponding time points in the total study population $(n=30)$.

\begin{tabular}{lcc}
\hline & $R$ & $p$ \\
\hline BL & 0.17 & $\mathrm{~ns}$ \\
3 hours & 0.51 & $<0.01$ \\
12 hours & 0.63 & $<0.01$ \\
Day 1 & 0.40 & 0.03 \\
Day 3 & 0.28 & $\mathrm{~ns}$ \\
Day 5 & 0.53 & $<0.01$ \\
Day 7 & 0.21 & $\mathrm{~ns}$ \\
Day 14 & 0.50 & $<0.01$ \\
\hline R: Sparma
\end{tabular}

$R$ : Spearman rho. BL: baseline. 


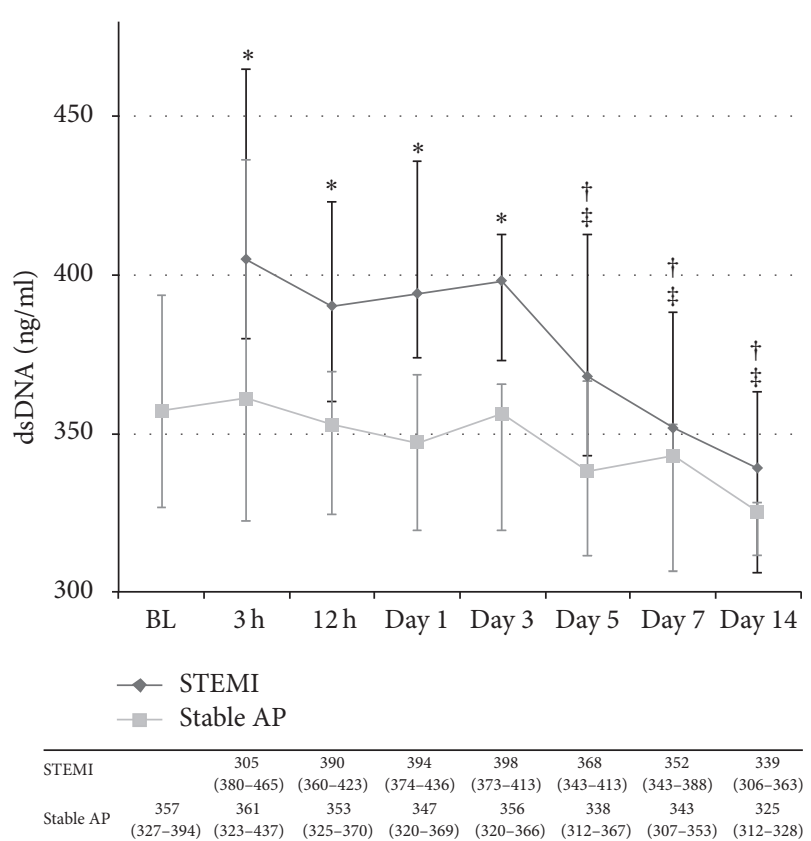

(a)

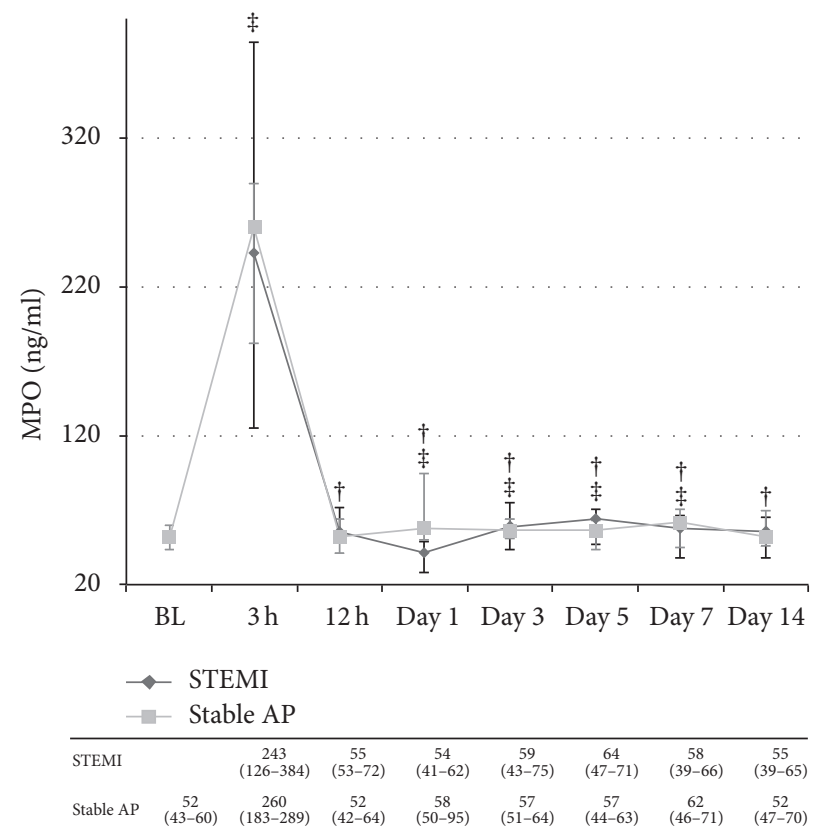

(c)

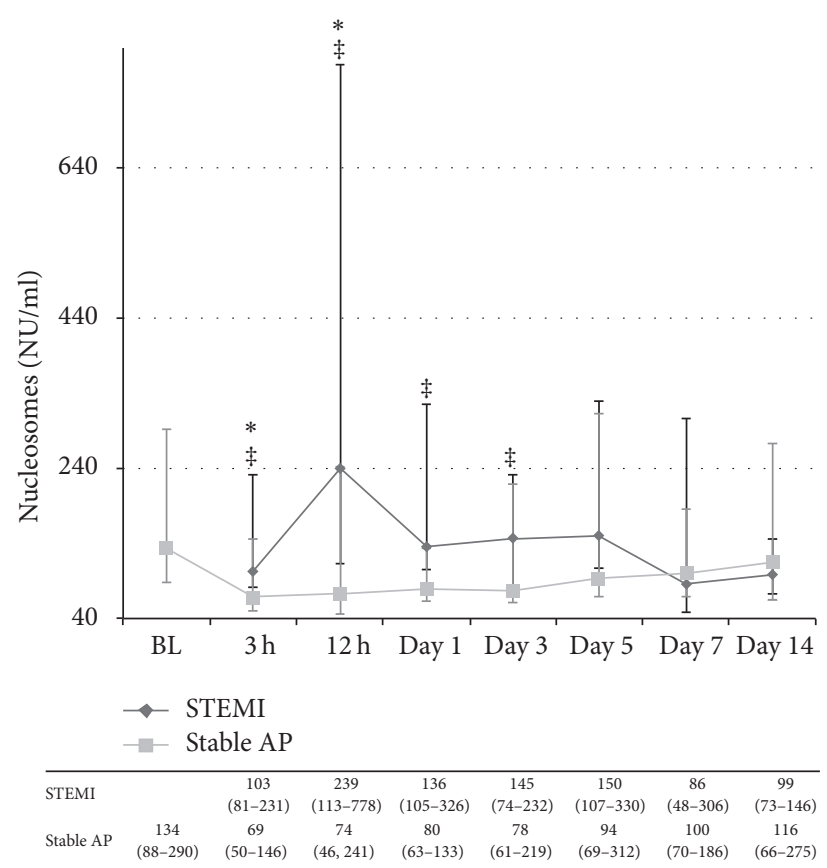

(b)

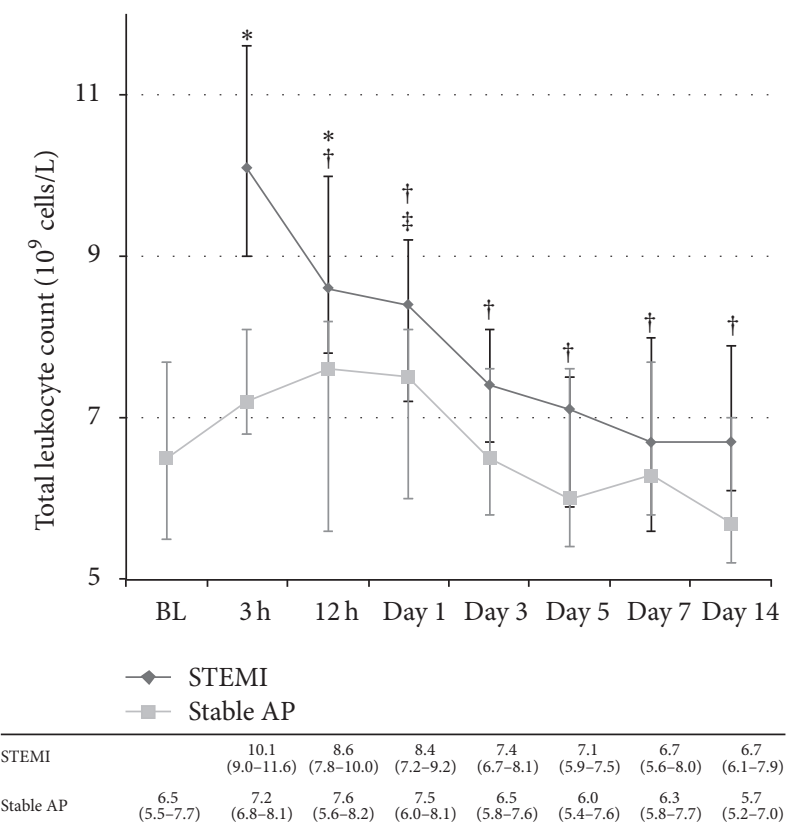

(d)

FIGURE 1: Time profiles of the NETs markers, myeloperoxidase, and leukocyte count. (a) Double-stranded deoxyribonucleic acid (dsDNA), (b) nucleosomes (DNA-histone complexes), (c) myeloperoxidase (MPO), and (d) total leukocyte count. The various time points ( $x$-axis) and levels ( $y$-axis) in the ST-elevation myocardial infarction (STEMI) and stable angina pectoris (AP) groups, as well as between- and withingroup comparisons. Values are given as median (25-75 percentiles). BL: baseline. ${ }^{*} p<0.05$ for between-group difference at the various time points. ${ }^{\dagger} p<0.05$ for within-group difference from 3 hours in the STEMI group. ${ }^{\ddagger} p<0.05$ for within-group difference from baseline in the stable AP group.

correlate significantly with either dsDNA or nucleosomes at any time point (data not shown).

3.4. Correlations with Total Leukocyte Count. In the total cohort, levels of dsDNA correlated positively with total leukocyte count at the majority of time points while the nucleosomes correlated to a lesser degree. Levels of MPO correlated with total leukocyte count only at day 14 (Table 3). The correlations were less obvious when analyzing the groups separately (data not shown), although the time profile of 
TABLE 3: Correlation between markers of NETs, MPO, and total leukocyte count at corresponding time points in the total study population $(n=30)$.

\begin{tabular}{|c|c|c|c|c|c|c|}
\hline & \multicolumn{2}{|c|}{ dsDNA } & \multicolumn{2}{|c|}{ Nucleosomes } & \multicolumn{2}{|c|}{ MPO } \\
\hline & $R$ & $p$ & $R$ & $p$ & $R$ & $p$ \\
\hline Baseline $(n=10)$ & 0.61 & ns & 0.13 & ns & 0.06 & ns \\
\hline 3 hours & 0.42 & 0.03 & 0.37 & 0.05 & -0.04 & ns \\
\hline 12 hours & 0.37 & 0.05 & 0.30 & ns & 0.36 & ns \\
\hline Day 1 & 0.22 & ns & 0.45 & 0.01 & 0.07 & ns \\
\hline Day 3 & 0.57 & $<0.01$ & 0.51 & $<0.01$ & 0.17 & ns \\
\hline Day 5 & 0.54 & $<0.01$ & 0.59 & $<0.01$ & 0.36 & ns \\
\hline Day 7 & 0.49 & $<0.01$ & 0.18 & ns & 0.09 & ns \\
\hline Day 14 & 0.39 & 0.04 & 0.21 & ns & 0.47 & 0.01 \\
\hline
\end{tabular}

$R$ : Spearman rho. BL: baseline.

total leukocyte count in both groups moderately imitated the pattern of dsDNA (Figure $1(\mathrm{~d})$ ).

3.5. Correlations with Myocardial Injury. Levels of dsDNA correlated positively with peak levels of troponin $\mathrm{T}$ and CKMB at day 5 ( $r=0.48, p=0.03$ for both), while day 5 and 7 levels correlated with infarct size assessed by MRI ( $r=0.61$, $p=0.01$ and $r=0.52, p \leq 0.04$, resp.) (Figures 2(a)-2(d)). Also, nucleosome levels at day 5 correlated with infarct size by MRI ( $r=0.58, p=0.02$ ) (Figure 2(e)), while levels of MPO were not correlated with any indices of myocardial injury. Either dsDNA, nucleosome, or MPO levels correlated with LVEF at any time point (data not shown).

\section{Discussion}

The main findings of this explorative, hypothesis generating study were as follows: (1) the two circulating surrogate markers of NETs, dsDNA and nucleosomes, were higher in patients with STEMI compared to patients with stable AP shortly after PCI, and levels were to a certain degree associated with indices of myocardial injury; (2) dsDNA and nucleosome levels decreased after PCI also in patients with stable AP, indicating that transient coronary ischemia might be associated with production of these NETs markers; (3) circulating levels of MPO did not reflect levels of dsDNA or nucleosomes and were not affected by the STEMI, but apparently by the PCI procedure itself.

Although circulating DNA has been reported to be higher in AMI than in both stable AP and healthy controls [11-15], no studies have to the best of our knowledge reported on the detailed time profile of NETs surrogate markers during STEMI and stable AP. The observed elevated levels of dsDNA and nucleosomes in patients with STEMI shortly after PCI are noticeable and may reflect enhanced neutrophil cell activation, myocardial cell necrosis with subsequent release of nuclear content, or a combination of more events. As strong correlations between dsDNA and nucleosome levels obtained in the subacute phase following PCI and infarct size measured by MRI after 6 weeks were observed, these potential markers of NETs could reflect myocardial cell necrosis directly. The possibility that they also represent neutrophil cell activation is nevertheless present, as strong correlations between circulating total leukocyte count and both dsDNA and nucleosome levels were observed at most time points and because the time profile of total leukocyte count imitated the time profile of dsDNA. Neutrophils have further previously been suggested to be the major source of circulating levels of both dsDNA and nucleosomes [10, 24]. As NETs probably hold prothrombotic properties like platelet activation [18], coagulation activation by factor XII and tissue factor, tissue factor pathway inhibitor (TFPI) suppression $[19,20]$ and inhibition of fibrinolysis [21], a role in coronary thrombosis would be plausible. As the present sample size was limited and the infarcts were relatively small, possible linkages between the assessed markers of NETs and coronary thrombosis need further exploration.

Elevated levels of dsDNA and nucleosomes shortly after PCI were not limited to the STEMI group, as decreasing levels of both markers also were observed in the stable AP group throughout the study period. In line with our observations, levels of both dsDNA and nucleosomes have previously been reported to associate with the severity of coronary atherosclerosis $[10,12]$, indicative of a link between AP with coronary ischemia and stimulated production of NETs (socalled NETosis). The pathophysiological properties of NETs in atherosclerosis and thus potentially also in AP have not been explored in detail, but have in murine models been suggested to include stimulation and migration of various innate and adaptive immune cells into the atherosclerotic plaque, partly through macrophage-mediated cytokine release [16, 25] and endothelial dysfunction mediated through metalloproteinases [26]. Given the prothrombotic properties of NETs, it would also be intriguing to speculate whether NETs participate in microthrombosis following intraplaque hemorrhage, a well-established cause of atherosclerotic plaque progression [27].

Levels of MPO, a well-known neutrophil granule protein and a proposed component of NETs [7, 28], were not reflected in neither dsDNA nor nucleosome levels. If dsDNA and nucleosomes indeed are part of NETs, this observation suggests that circulating MPO were mainly not NETs-derived. The lack of association between MPO and 


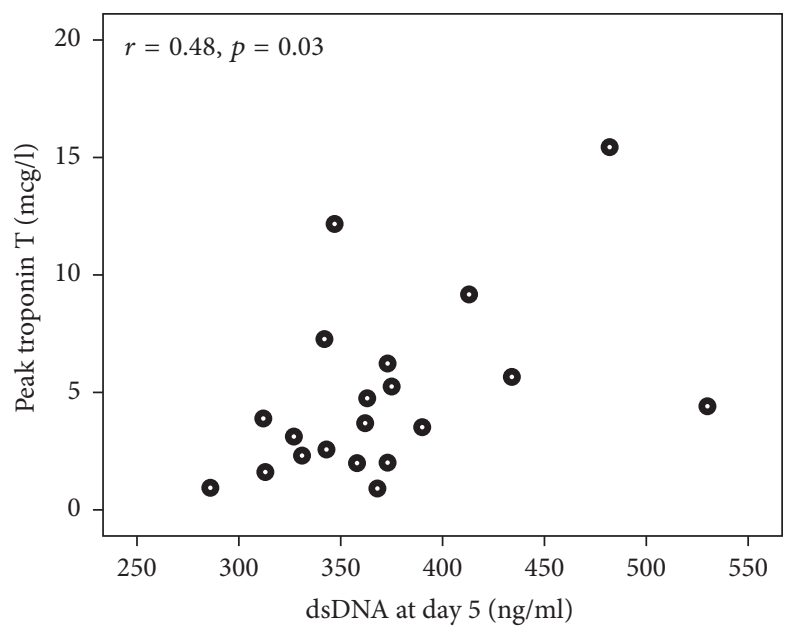

(a)

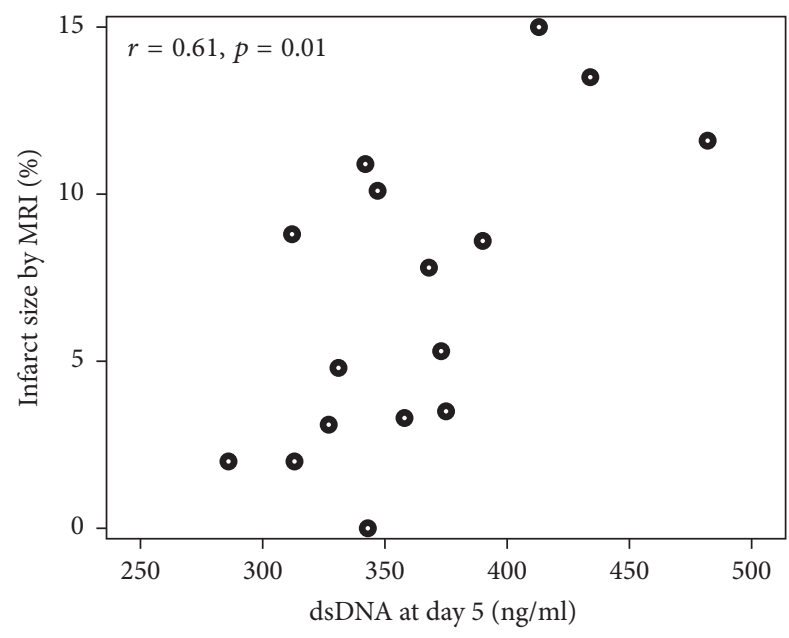

(c)

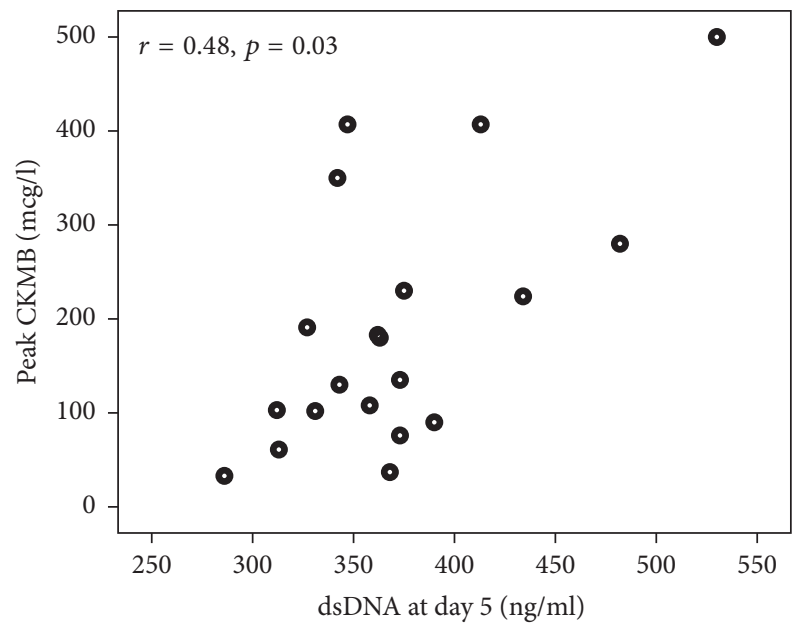

(b)

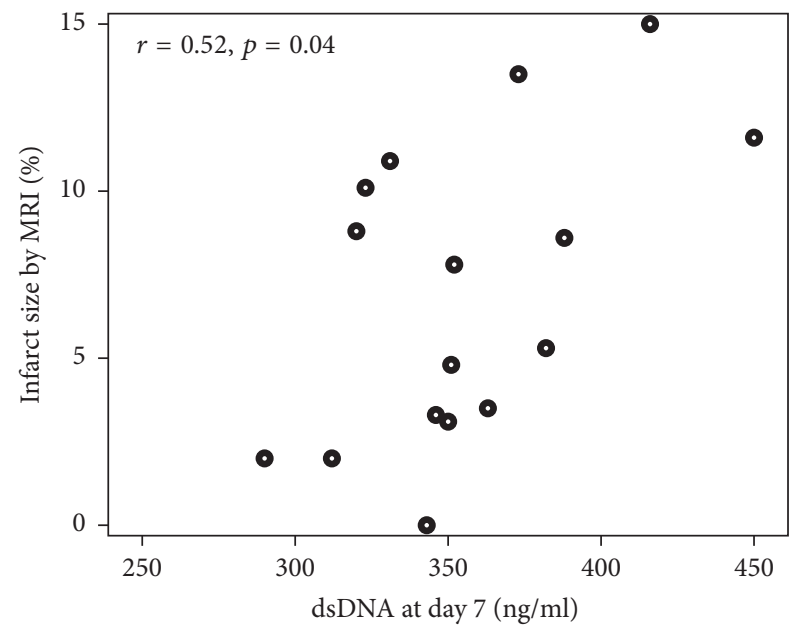

(d)

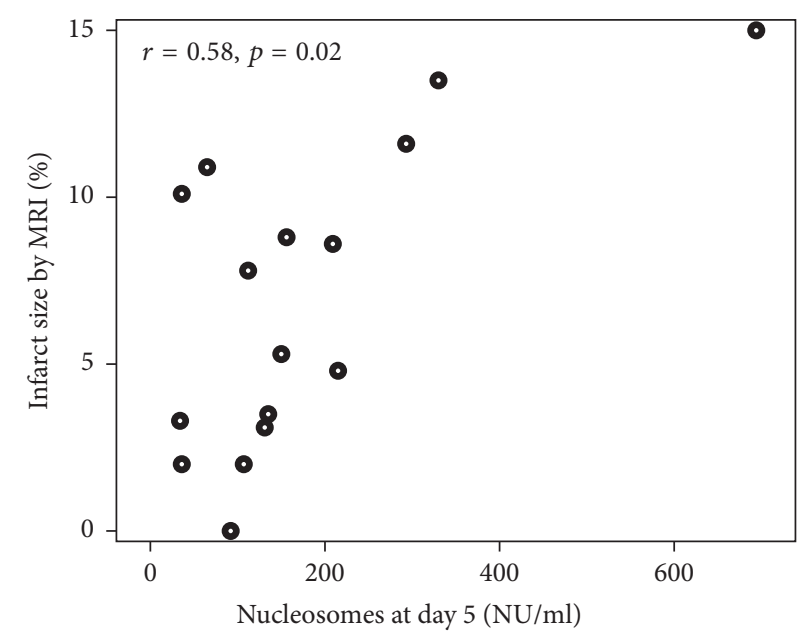

(e)

FIGURE 2: Correlations between NETs markers and indices of infarct size. dsDNA: double-stranded deoxyribonucleic acid. CKMB: creatine kinase MB. MRI: magnetic resonance imaging. 
dsDNA/nucleosomes is consistent with that of another neutrophil granule protein, PTX3 [23], suggesting that despite release from neutrophil granules upon neutrophil cell activation, these two proteins did not enter circulating blood simultaneously with dsDNA and nucleosomes and did thus presumably not reflect NETosis in this cohort. Further, while MPO levels were not influenced by the STEMI, increased levels shortly after PCI in the stable AP group may be indicative of a direct effect of the PCI procedure per se. The mechanistic explanation for this apparent PCI-effect is unclear. Lastly, MPO was not correlated with any indices of myocardial injury or left ventricular function in contrast to the previously reported adverse properties of MPO in left ventricular remodeling after AMI [29, 30]. Again, limited sample size and small infarct sizes may have influenced these results.

The discovery of markers of NETs in atherothrombosis is of particular interest as NETs could be subject to therapeutic manipulations, that is, through administration of enzymes able to dissolve the NET structure like deoxyribonucleases (DNAses) or through inhibition of peptidylarginine deiminase 4 (PAD4), an essential enzyme in NETosis. Although in vivo experiments in humans have not been performed so far, adding DNAse to standard thrombolysis (with t-PA) has been shown to accelerate thrombus lysis in human ex vivo thrombi [8] and to reduce myocardial no-flow area, infarct size, and ischemia-reperfusion-induced left ventricular remodeling in rats [31]. Likewise, PAD4 inhibition has been shown to reduce NETosis in both murine and human neutrophils and further to directly interfere with atherosclerotic burden in mice [17, $32,33]$.

\section{Limitations}

Beyond limited sample size and small infarctions as previously described, the hypothesis generating nature of this study carries methodological limitations, as the high CV for the nucleosome analysis. To what extent circulating levels of dsDNA and nucleosomes are specific for NETs is further not clear. Moreover, aspirin, heparin, and statins have all been reported to suppress or destabilise NETs in murine and human models [18, 34-37], which could explain falling levels of dsDNA and nucleosomes in both groups, as well as lower levels generally in the AP group. Finally, as differential counts for neutrophils, the major source of dsDNA [24], were not available, our analyses are based on total leukocyte counts. These are though to a high degree representative for circulating neutrophils.

\section{Conclusions}

High levels of the NETs markers dsDNA and nucleosomes were observed in patients with STEMI shortly after revascularisation and did partly reflect infarct size. The decline over time, also observed in AP patients, may indicate that transient coronary ischemia also stimulates release of NETs markers. Together, these observations may imply roles for NETs in atherothrombosis.

\section{Disclosure}

Study identification number (Regional Committee for Medical Research Ethics) is 126-03032.

\section{Competing Interests}

The authors declared no competing interests.

\section{Authors' Contributions}

Ragnhild Helseth, Svein Solheim, Harald Arnesen, Ingebjørg Seljeflot, and Trine Baur Opstad all contributed significantly to the conceptualization, methodology, visualization, and writing. Trine Baur Opstad also performed the laboratory analyses, while Ragnhild Helseth performed the formal analyses.

\section{Acknowledgments}

The authors thank Pavel Hoffmann, Torstein Jensen, and Haakon K. Grøgaard for taking part in the inclusion of patients.

\section{References}

[1] S. Chia, J. T. Nagurney, D. F. M. Brown et al., "Association of leukocyte and neutrophil counts with infarct size, left ventricular function and outcomes after percutaneous coronary intervention for st-elevation myocardial infarction," The American Journal of Cardiology, vol. 103, no. 3, pp. 333-337, 2009.

[2] L. Guasti, F. Dentali, L. Castiglioni et al., "Neutrophils and clinical outcomes in patients with acute coronary syndromes and/or cardiac revascularisation. A systematic review on more than 34,000 subjects," Thrombosis and Haemostasis, vol. 106, no. 4, pp. 591-599, 2011.

[3] R. Latini, A. P. Maggioni, G. Peri et al., "Prognostic significance of the long pentraxin PTX3 in acute myocardial infarction," Circulation, vol. 110, no. 16, pp. 2349-2354, 2004.

[4] D.-H. Lee, H.-K. Jeon, J.-H. You et al., "Pentraxin 3 as a novel marker predicting congestive heart failure in subjects with acute coronary syndrome," Korean Circulation Journal, vol. 40, no. 8, pp. 370-376, 2010.

[5] S. Baldus, C. Heeschen, T. Meinertz et al., "Myeloperoxidase serum levels predict risk in patients with acute coronary syndromes," Circulation, vol. 108, no. 12, pp. 1440-1445, 2003.

[6] D. A. Morrow, M. S. Sabatine, M.-L. Brennan et al., "Concurrent evaluation of novel cardiac biomarkers in acute coronary syndrome: myeloperoxidase and soluble CD40 ligand and the risk of recurrent ischaemic events in TACTICS-TIMI 18," European Heart Journal, vol. 29, no. 9, pp. 1096-1102, 2008.

[7] V. Brinkmann, U. Reichard, C. Goosmann et al., "Neutrophil extracellular traps kill bacteria," Science, vol. 303, no. 5663, pp. 1532-1535, 2004.

[8] A. Mangold, S. Alias, T. Scherz et al., "Coronary neutrophil extracellular trap burden and deoxyribonuclease activity in ST-elevation acute coronary syndrome are predictors of STsegment resolution and infarct size," Circulation Research, vol. 116, no. 7, pp. 1182-1192, 2015. 
[9] J. Riegger, R. A. Byrne, M. Joner et al., "Histopathological evaluation of thrombus in patients presenting with stent thrombosis. A multicenter European study: a report of the prevention of late stent thrombosis by an interdisciplinary global European effort consortium," European Heart Journal, vol. 37, no. 19, pp. 1538.11549, 2016.

[10] J. I. Borissoff, I. A. Joosen, M. O. Versteylen et al., "Elevated levels of circulating DNA and chromatin are independently associated with severe coronary atherosclerosis and a prothrombotic state," Arteriosclerosis, Thrombosis, and Vascular Biology, vol. 33, no. 8, pp. 2032-2040, 2013.

[11] C. P.-Y. Chang, R.-H. Chia, T.-L. Wu, K.-C. Tsao, C.-F. Sun, and J. T. Wu, "Elevated cell-free serum DNA detected in patients with myocardial infarction," Clinica Chimica Acta, vol. 327, no. 1-2, pp. 95-101, 2003.

[12] M. Cui, M. Fan, R. Jing et al., "Cell-free circulating DNA: a new biomarker for the acute coronary syndrome," Cardiology, vol. 124, no. 2, pp. 76-84, 2013.

[13] A. Shimony, D. Zahger, H. Gilutz et al., "Cell free DNA detected by a novel method in acute ST-elevation myocardial infarction patients," Acute Cardiac Care, vol. 12, no. 3, pp. 109-111, 2010.

[14] A. Destouni, C. Vrettou, D. Antonatos et al., "Cell-free DNA levels in acute myocardial infarction patients during hospitalization," Acta Cardiologica, vol. 64, no. 1, pp. 51-57, 2009.

[15] D. Antonatos, S. Patsilinakos, S. Spanodimos, P. Korkonikitas, and D. Tsigas, "Cell-free DNA levels as a prognostic marker in acute myocardial infarction," Annals of the New York Academy of Sciences, vol. 1075, pp. 278-281, 2006.

[16] A. Warnatsch, M. Ioannou, Q. Wang, and V. Papayannopoulos, "Neutrophil extracellular traps license macrophages for cytokine production in atherosclerosis," Science, vol. 349, no. 6245, pp. 316-320, 2015.

[17] J. S. Knight, W. Luo, A. A. O’Dell et al., "Peptidylarginine deiminase inhibition reduces vascular damage and modulates innate immune responses in murine models of atherosclerosis," Circulation Research, vol. 114, no. 6, pp. 947-956, 2014.

[18] C. Kaiser, M. Koranda, B. Kitzler et al., "Belowground carbon allocation by trees drives seasonal patterns of extracellular enzyme activities by altering microbial community composition in a beech forest soil," New Phytologist, vol. 187, no. 3, pp. 843-858, 2010.

[19] M.-L. von Brühl, K. Stark, A. Steinhart et al., "Monocytes, neutrophils, and platelets cooperate to initiate and propagate venous thrombosis in mice in vivo," Journal of Experimental Medicine, vol. 209, no. 4, pp. 819-835, 2012.

[20] S. Massberg, L. Grahl, M.-L. von Bruehl et al., "Reciprocal coupling of coagulation and innate immunity via neutrophil serine proteases," Nature Medicine, vol. 16, no. 8, pp. 887-896, 2010.

[21] S. H. C. Mai, M. Khan, D. J. Dwivedi et al., "Delayed but not early treatment with dnase reduces organ damage and improves outcome in a murine model of sepsis," Shock, vol. 44, no. 2, pp. 166-172, 2015.

[22] S. Solheim, H. K. Grøgaard, P. Hoffmann, H. Arnesen, and I. Seljeflot, "Inflammatory responses after percutaneous coronary intervention in patients with acute myocardial infarction or stable angina pectoris," Scandinavian Journal of Clinical and Laboratory Investigation, vol. 68, no. 7, pp. 555-562, 2008.

[23] R. Helseth, S. Solheim, T. Opstad, P. Hoffmann, H. Arnesen, and I. Seljeflot, "The time profile of pentraxin 3 in patients with acute ST-elevation myocardial infarction and stable angina pectoris undergoing percutaneous coronary intervention," Mediators of Inflammation, vol. 2014, Article ID 608414, 5 pages, 2014.

[24] T. J. Gould, T. T. Vu, L. L. Swystun et al., "Neutrophil extracellular traps promote thrombin generation through plateletdependent and platelet-independent mechanisms," Arteriosclerosis, Thrombosis, and Vascular Biology, vol. 34, no. 9, pp. 19771984, 2014.

[25] Y. Döring, H. D. Manthey, M. Drechsler et al., "Auto-antigenic protein-DNA complexes stimulate plasmacytoid dendritic cells to promote atherosclerosis," Circulation, vol. 125, no. 13, pp. 1673-1683, 2012.

[26] C. Carmona-Rivera, W. Zhao, S. Yalavarthi, and M. J. Kaplan, "Neutrophil extracellular traps induce endothelial dysfunction in systemic lupus erythematosus through the activation of matrix metalloproteinase-2," Annals of the Rheumatic Diseases, vol. 74, no. 7, pp. 1417-1424, 2015.

[27] P. Libby and P. Theroux, "Pathophysiology of coronary artery disease," Circulation, vol. 111, no. 25, pp. 3481-3488, 2005.

[28] R. K. Schindhelm, L. P. van der Zwan, T. Teerlink, and P. G. Scheffer, "Myeloperoxidase: a useful biomarker for cardiovascular disease risk stratification?" Clinical Chemistry, vol. 55, no. 8, pp. 1462-1470, 2009.

[29] A. T. Askari, M.-L. Brennan, X. Zhou et al., "Myeloperoxidase and plasminogen activator inhibitor 1 play a central role in ventricular remodeling after myocardial infarction," Journal of Experimental Medicine, vol. 197, no. 5, pp. 615-624, 2003.

[30] N. Vasilyev, T. Williams, M.-L. Brennan et al., "Myeloperoxidase-generated oxidants modulate left ventricular remodeling but not infarct size after myocardial infarction," Circulation, vol. 112, no. 18, pp. 2812-2820, 2005.

[31] L. Ge, X. Zhou, W.-J. Ji et al., "Neutrophil extracellular traps in ischemia-reperfusion injury-induced myocardial no-reflow: therapeutic potential of DNase-based reperfusion strategy," American Journal of Physiology. Heart and Circulatory Physiology, vol. 308, no. 5, pp. H500-H509, 2015.

[32] H. D. Lewis, J. Liddle, J. E. Coote et al., "Inhibition of PAD4 activity is sufficient to disrupt mouse and human NET formation," Nature Chemical Biology, vol. 11, no. 3, pp. 189-191, 2015.

[33] G. P. Fadini, L. Menegazzo, M. Rigato et al., "NETosis delays diabetic wound healing in mice and humans," Diabetes, vol. 65, no. 4, pp. 1061-1071, 2016.

[34] E. Tarantino, P. Amadio, I. Squellerio et al., "Role of thromboxanedependent platelet activation in venous thrombosis: aspirin effects in mouse model," Pharmacological Research, vol. 107, pp. 415-425, 2016.

[35] D. M. Sayah, B. Mallavia, F. Liu et al., "Neutrophil extracellular traps are pathogenic in primary graft dysfunction after lung transplantation," American Journal of Respiratory and Critical Care Medicine, vol. 191, no. 4, pp. 455-463, 2015.

[36] M. J. Lapponi, A. Carestia, V. I. Landoni et al., "Regulation of neutrophil extracellular trap formation by anti-inflammatory drugs," Journal of Pharmacology and Experimental Therapeutics, vol. 345, no. 3, pp. 430-437, 2013.

[37] C. W. Kessinger, J. W. Kim, P. K. Henke et al., "Statins improve the resolution of established murine venous thrombosis: reductions in thrombus burden and vein wall scarring," PLoS ONE, vol. 10, no. 2, Article ID e0116621, 2015. 


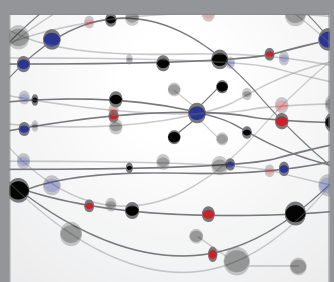

The Scientific World Journal
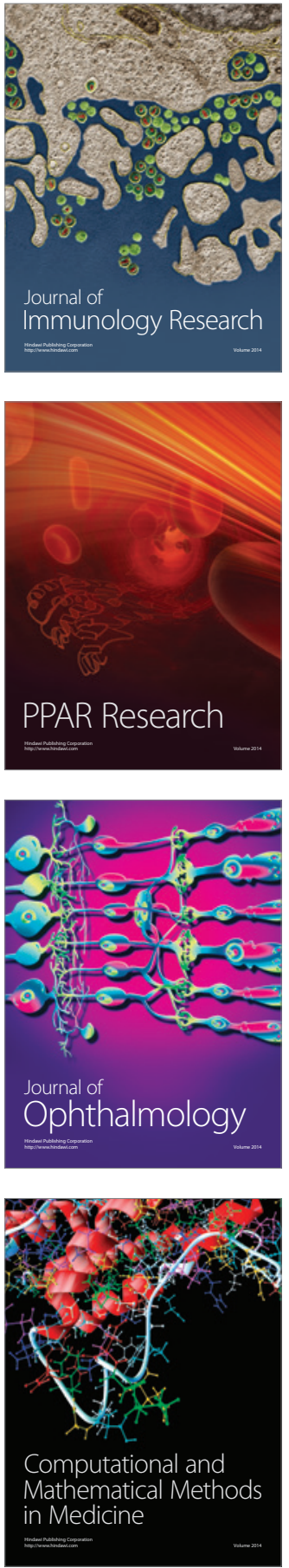

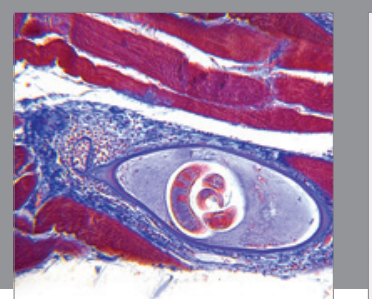

Gastroenterology Research and Practice

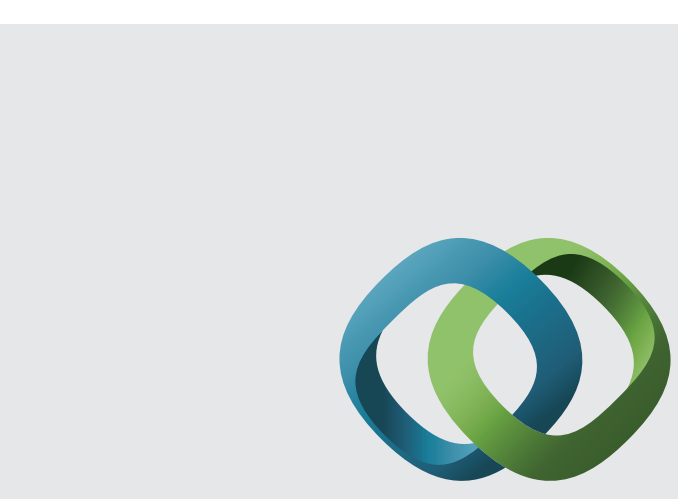

\section{Hindawi}

Submit your manuscripts at

http://www.hindawi.com
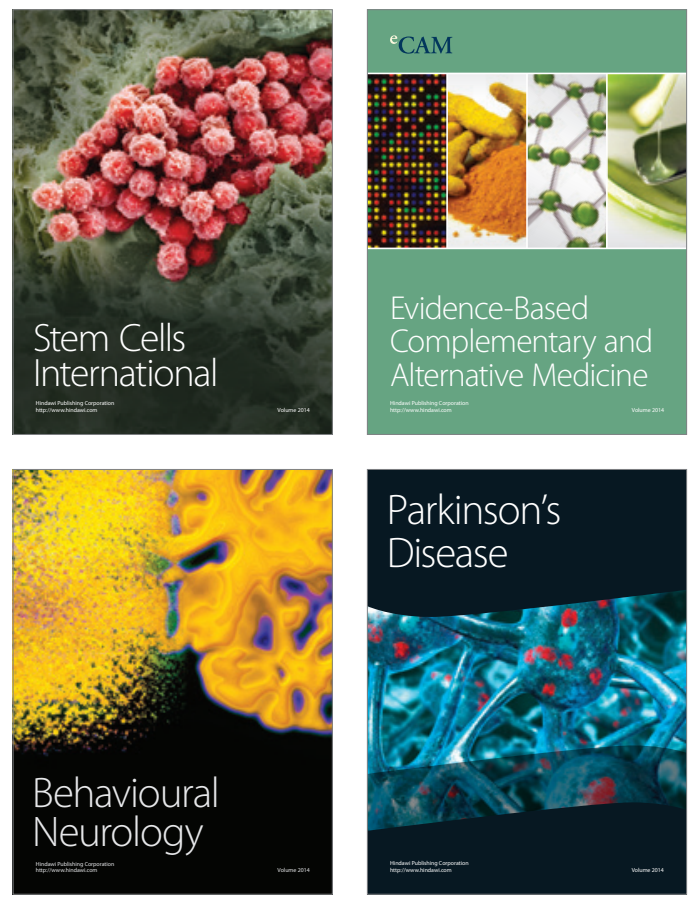
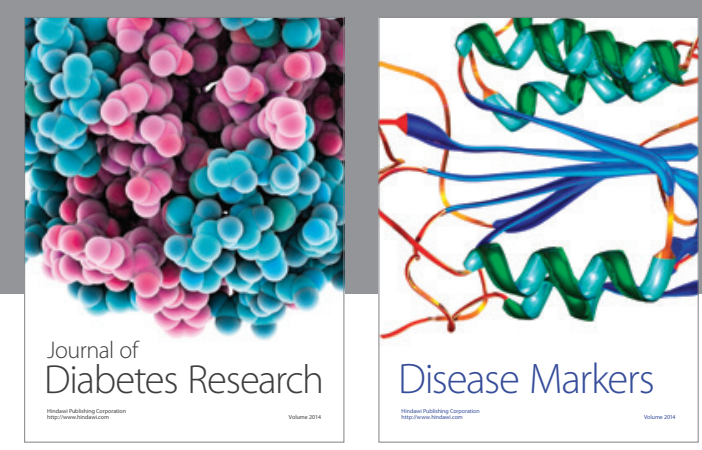

Disease Markers
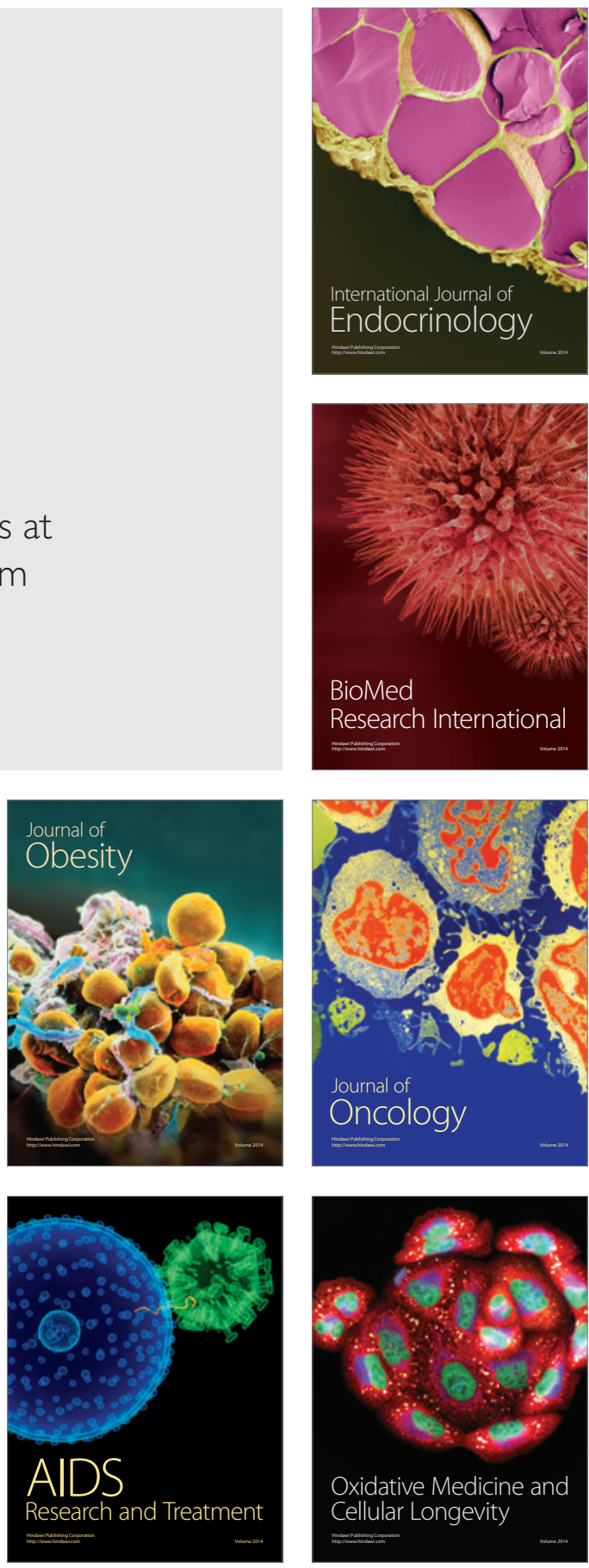